\title{
Digital Libraries: What users want?
}

\author{
Elahe Kani-Zabihi, Gheorghita Ghinea and Sherry Y. Chen \\ School of Information Systems, Computing and Mathematics \\ Brunel University \\ Uxbridge, UB8 3PH, UK \\ \{Elahe.Kani, George.Ghinea, Sherry.Chen\}@brunel.ac.uk
}

\begin{abstract}
Purpose - The purpose of this study was to determine user suggestions for Digital Libraries' functionality and features.

Design/methodology/approach - A survey was conducted as part of this study, in which users' suggestions for Digital Libraries were solicited, as well as their ranking opinions on a range of suggested Digital Library features.

Findings - The study revealed that, regardless of users' Information Technology (IT) backgrounds, their expectations of Digital Libraries' functionality are the same. However, based on users' previous experiences with Digital Libraries, their requirements with respect to specific features may change.

Practical implications - Involving users in Digital Library design should be an integral step in the process of building a Digital Library - in addition to the classic roles of evaluation and testing.

Originality/value - In previous Digital Library user studies, users were involved implicitly (e.g. observed) or explicitly (e.g. diary notes). However, they were never asked to suggest Digital Library features or functionalities, as this was left to usability and domain experts. This study approached Digital Library design from a new perspective, giving users an opportunity to express their suggestions on future functionality and features of Digital Libraries. Moreover, in contrast to previous work, this study has explicitly taken into account the IT abilities of those interacting with a Digital Library.

Keywords - Digital Library, Human Computer Interaction, user-centred design, user study

Paper type - Research Paper
\end{abstract}

\section{Introduction}

In implementing a Digital Library (DL), users are often not involved in the design cycle, as experts from library science, computer science and Human Computer Interaction (HCI) all participate in specifying the system design (Witten et al., 2001). Indeed, DLs are built as a response to the needs of a particular community, and rarely involve people with any previous domain experience (Suleman and Fox, 2001). DLs are then evaluated by undertaking experiments that usually employ a small group of users.

DLs have addressed many design issues, notably the "tension" between DL designers and authors and librarians ("information suppliers") of DLs (Theng et al., 2000). Furthermore, DLs currently provide inadequate support for activities, such as use of the system owing to a lack of familiarity and learning as well as the lack of space for 
users to work and collaborate. Information in DLs can be viewed as having a form of a "quality stamp", which restricts DL users as to the content they can access, thus creating the need to have "sophisticated information skills" (Blandford, 2004). With this in mind, DLs should be designed according to these needs. A focus group should be employed to tackle these problems and provide an understanding of how users work with and use information and how these observations can be fed into the design of a DL to enhance, rather than obstruct, the experience of using information (Theng et al., 2000).

Having a better understanding of user behaviours, needs, and user oriented design (Blandford and Buchanan, 2003) would enable designers to appreciate what end users feel about using DLs and what changes would be needed to meet their needs. It is for this reason that the future of DLs, just like any other information system, is very much dependent on its users. DL use will be dictated by people, so it makes sense to know more about them and their needs. To this end, the study presented in this paper investigates what expectations users have from DLs. Accordingly, the structure of this paper is as follows: section 2 presents related work in the area of DLs, with section 3 detailing the experiments undertaken as part of our study, results are presented in section 4 , with conclusions as possibility for future work being identified in section 5 .

\section{Background}

DLs are given different names by the various stakeholders working with them. Thus, librarians refer to them as 'databases' and people in arts and humanities name them 'electronic archives' (Adams and Blandford, 2002). In the UK and Western Europe, DLs are referred to as 'digital surrogates', being regarded as substitutes for traditional libraries in their role of 'collections of validated and structured information' (Blandford, forthcoming).

One definition for a DL would be that: a DL is a collection of services and "information objects" which are available digitally. Information objects can be defined as anything in a digital format such as books, journal articles and sounds, since DLs organise and present information objects to users and support them in dealing with these objects (Leiner, 2005).

Lang (1998) describes that the goal of a DL is to improve access for all users: "The digital library is the widely accepted term describing the use of digital technologies to acquire, store, preserve and provide access to information and material originally published in digital form or digitised from existing print, audio-visual and other forms".

The above definitions all convey the same meaning, which reveal that a DL is a source of information, in different forms e.g. text or audio, which is stored digitally. Moreover, given the proliferation of communication technologies such as the Internet, it comes as no surprise that, in a modern context, DLs also represent a networked resource. Thus, for the purposes of the research described in this paper, the definition that shall be adopted is that $a$ DL is a networked repository of digital content. 
The definition of DLs implicitly conveys the advantages of DLs. For example, DLs have changed the way humans interact with information, as the requested information can be accessed from anywhere at anytime, as long as there is an Internet connection available and the user has an appropriate access device. Moreover, in so doing, DLs have introduced learning, and especially distance learning, to new dimensions. (Blandford, forthcoming). Another advantage of DLs is that, as opposed to traditional libraries, which have a limited storage space, DLs have the advantages of saving physical space by holding all information digitally on a server. Indeed, DLs can always extend their storage space, as long as relatively inexpensive computer memory is available. What is also interesting is that there need not be a one-to-one mapping between a DL and a traditional library, as the information contained in a DL could be distributed geographically in separate sites (Slonim and BaronLaurie, 1994).

Given the benefits that DLs have compared with traditional libraries, there is no doubt that DL use will experience high growth rates in upcoming years. Given this trend and the ubiquity of the Internet, it seems natural to recognise that DL use will not be restricted to specialist users, and that a variety of users with different Information Technology (IT) backgrounds will be accessing DLs in the future. DL designers must therefore design them in a way which makes DLs easy to understand and learn, irrespective of the particular IT skills of the user.

Designers are aware that DL utility is measured by getting more satisfaction from users. Hence when considering making DL services more helpful, users must be the focus of attention, as designers can learn more about their needs and try to fulfil them. For example, in designing user interfaces, designers can find out from their users whether DLs interfaces are designed in a user friendly manner. However, while user considerations have been integrated in the design of DLs from a usability perspective (Blandford et al., 2004), it is to be remarked that users are rarely, if even, consulted for their opinions on DL functionality and features.

This is not to say, though, that users have not been involved in existing studies on DL design. Thus, one study on online (database) searching experiences, investigated by Hsieh-Yee (1993) involved two groups of users. The first was made up of 32 professional online searchers (who had at least one year of search experience or had taken courses on online searching), whilst the second comprised 30 novice searchers (who had little or no search experience and had not taken any courses on online searching). This study investigated how people use their search methods to search an online database and also how their search experience and subject knowledge had an effect on their searching methods. For this, users were given two test tasks in the subject area of each searcher group, namely to find some documents on online databases.

Hsieh-Yee (1993) used three approaches for data collections: think out loud, transactional logs and observation. Users were asked to think out loud while a search was done, and their interaction with the system was recorded by a communication program. In addition, a research assistant observed users behaviour during their experiments. The observer helped in collecting useful information to give a context for the transaction logs. The conclusion of the study was that the subject expertise and search experience have an effect in the search method employed by users. 
Another approach for collecting data from users is by interviewing them. This method was used by Fox et al. (1993), in the Envision project, who interviewed users and experts in three areas: library, information and computer science. The aim of this project was to build a "user-centred database", by interviewing users about potential decisions on system functionality. The project focused on 12 participants who were professionals in the area of computer science and information retrieval. Participants were questioned on four topics:

- current information retrieval practices

- current information dissemination practices

- desired information retrieval and manipulation capabilities

- demographic data

The result of this project was a "usable prototype digital library" and a set of nine principles for DLs. Of particular interest to our study was the $8^{\text {th }}$ Principle, which declared that "a user-centred development approach should be adopted" and that the design focus should be on users, as otherwise DLs would not communicate effectively and efficiently with their users.

Conversely, Twidale and Nichols (1998) collected their data implicitly from users by observing them while working. They observed librarians' collaboration in the library, while using the library's Online Public Access Catalogue (OPAC), and also whilst doing other library activities. The main conclusion of this study was that users achieve their goals through collaboration, which has an important role in their accomplishment. Even with well designed library systems, users have difficulties to find their information. They may, however, be helped by a member of library staff that may not have their subject expertise. Hence the computer interface in a library system plays an important role by being a conversational resource between an information seeker and a library staff.

In related work, Stelmaszewska and Blandford (forthcoming) undertook a study in order to better understand people's interactions and their needs in the context of a traditional, physical library. They examined 12 computer science students and 2 part time tutors in computer science while they were using a physical library. The results of this study showed that BLAH BLAH

From a different perspective, a study carried by Fields et al. (2004) observed librarians while helping students who did not have sufficient skills to find the information they need using DLs. For this, two librarians were video taped and were asked to think out loud. The conclusion given from this study is that expert users of DL are more successful at finding information than non-experts in DLs, thus supporting our view that DL design has to take into account the IT skills of the user.

In an extensive review of user involvement in the constructions of DLs, Bryan-Kinns and Blandford (2000) identified the following types of user-based data gathering techniques in DLs:

1- Diary Studies: People were asked to keep daily notes.

2- Questionnaires: On-line form filling.

3- Observation: Observing people while using systems.

4- Usability Testing: Experimental design. 


\section{5- Focus Groups \\ 6- Interviews \\ 7- Transaction Logs}

It is interesting, though, to remark that of the 18 studies reviewed, all focused on computing user interfaces and search techniques, with none considering what people actually require of DLs, and how they might wish to use them.

In work more closely related to ours, Theng et al. (2000) undertook a user study in which 45 participants were asked for their opinion and suggestions on DLs. These participants were third year computer science undergraduate students split into three equal-size groups, with each group evaluating one of three DLs:

- ACM Digital Library (ACMDL)

- Networked Computer Science Technical Reference Library (NCSTRL)

- New Zealand Digital Library (NZDL)

A questionnaire was given to each user group, soliciting opinions on the design, structure, and purpose of DLs. Users were also asked to suggest design features for DLs. However, all participants were classified as "expert users" since they had an understanding of design and evaluation issues in interactive systems. The main results of the study showed that $74 \%$ of participants agreed that physical libraries should be replaced with DLs, and that $73 \%$ of users experienced disorientation when using the three DLs, since the DLs provided few, if any, useful cues to aid users in their tasks. Beside the questionnaire, participants were also asked to evaluate the three DLs by employing created usability heuristics. With a few exceptions, the usability of the ACMDL was found to be lower than that of the NCSTRL and NZDL; however, the usability values seemed to converge to a uniform pattern, when the number of participants increased.

In a similar vein, Stelmaszewska et al. (2001) also used computer scientists in a user study. They ran an experiment to explore users' interaction with DLs, in which a video-based observational study with think-aloud commentary was used for gathering data, followed by a short interview with participants. Participants were four $\mathrm{PhD}$ students and one member of the academic staff, all of whom were computer scientists. These were asked to find an article that would help them in their current research. From this experiment the authors concluded that in terms of the Interaction Framework (Bryan-Kinns et al., 2000), important issues in DLs are familiarity, blind alleys and serendipity.

A recent study (Zhang and Marchinonini, 2005) on browsing and searching large information collections involved users in the searching and browsing of video DLs, using different techniques to compare effectiveness, efficiency and user satisfaction. Two different interfaces were compared in this study: one was a new interface designed by Zhang and Marchionini called the Relation Browser ++ (RB++), while the other was a traditional form filling interface. Data collected from users were: the time spent finishing each task; error rate for each task; rating on their satisfaction; ratings on the usability of each interface; and finally an open question about users' opinions and preferences for two interfaces. Users' responses to this experiment were 
used to evaluate the designed interfaces. The results showed that users were more satisfied with $\mathrm{RB}++$ than the traditional form filling interface.

With the exception of Theng et al (2000), and Zhang and Marchionini (2005), in all the above user studies, users were either involved implicitly e.g. observed, or explicitly e.g. diary notes, to find out what they do during their interactions with DLs, with Zhang and Marchionini (2005) taking one step further by actually asking users their opinions with respect to different types of interfaces. However, users were not given any opportunity to discuss their understandings of DLs. Although Theng et al (2000) did ask users to comment on the purpose of DLs and to suggest design features for DLs, all these users were to some extent designers and classified as experts users. Therefore they were aware of design and evaluation issues within interactive systems, with the study resulting in an incomplete picture of user expectations with respect to DLs. Moreover, the study itself concluded that to get a broader understanding of the purpose of DLs, there is a need for an extensive study employing a variety of users of DLs.

There is therefore still a gap between what users want and what is available to them in DLs. The purpose of the study described in the present paper is to address this gap, by inclusively involving users with a wide spectrum of IT abilities and soliciting their opinions on what the main focus of DLs' requirements should be so, that their expectations are satisfied.

\section{Experiment}

As mentioned earlier, understanding users is very important in the early stage of the DL design process. We have therefore undertaken an experiment to find out the type of interactions and features that users prefer to have in a DL. In order to make sure users are acquainted with DLs, they were asked to use two DLs: Science Direct (www.sciencedirect.com) and the Classical Music Library (http://www.alexanderstreetpress.com/products/clmu.htm), as they are respectively text and audio oriented, thus exemplifying a basic variety of media choices. Science Direct has a wide range of journals and articles, in a various fields, and is used by most researchers. The Classical Music Library is also a good example of a media DL that allows users to research and listen to classical music.

\section{Participants}

We were not interested in a particular category of users; instead we polled users with a variety of IT knowledge backgrounds. A total of 48 users participated in our experiment; these were grouped into three main categories (novice, intermediate and advanced), with an equal number of users in each. In order to determine in which particular category a user belonged to, each participant in our study had to complete a questionnaire (Table I).

Participants, who answered YES to first three questions and NO to the rest, were categorised as being in the novice category; if participants answered YES to any three of the above questions in additions to first three questions they were deemed to be in the intermediate category. Further answering YES to either question 9 or 10, in addition to first eight questions made a user belong to the advanced category. 
take in Table I

\section{Procedure}

Our experiment was divided into three main parts, the first of which was a familiarisation with DLs. Accordingly, as mentioned above, in our study we used two DLs. In order to ensure that participants across the range of abilities had at least the same exposure to DLs, they were given the URLs of the two DLs and were told that they had 10 minutes (per DL) to familiarise themselves with the features, content, layout and feel of each, as they saw fit. They were explained that if they felt they were comfortable using the DL before the 10 minutes allocated for familiarisation had elapsed, they could start the second half of the experiment earlier, although there was no pressure put on them to do so by the experimenter.

Once the familiarisation was compete, the second part of our experiment involved users accomplishing a set of tasks. Thus, the two groups of tasks were:

\section{Science Direct:}

1. Search for an article with the title: A distributed systems approach to secure Internet mail.

2. Search for a journal named: Computer Communications. Then make a note of all articles in volume 29 of that journal.

3. Set up an alert so you can receive updated articles on computer science by email.

\section{Classical Music Library:}

1. Search for a track by an artist called Sarah Clarke. Make a note of the instrument that she used. Then add the track to 'My Playlists'.

2. Search for other tracks played by artists using the same instrument and add two of them to 'My Playlists'.

3. View the content of 'My Playlists' and play the tracks it contains.

Users were explained that they would not be judged on how fast or efficiently they performed the tasks, but rather that the purpose of the exercise was to give them additional exposure to each of the two DLs. No aid was offered to participants to accomplish the tasks.

The last part of the experiment involved users replying to a questionnaire soliciting their views on DLs. Accordingly we administered a questionnaire soliciting both qualitative and quantitative data, via the use of both structured and open-ended questions. The questionnaire itself contained two parts, the first of which solicited their opinions on the features that they would prefer that DLs contain. The second half of the questionnaire was itself comprised of two parts. In the first, users were asked to prioritise 5 given requirements of DLs (Table II) which the academic literatures Blandford (2004), D'Alessandro (2005), Blandford (forthcoming), has identified as being relevant for the construction of DLs. 
Table II - List of requirements suggested to participants

take in Table II

Finally, we also asked users to express their opinions on a 5-point scale (not important, a little important, moderately important, very important, and extremely important) with respect to 19 of our own suggestions for future DLs (Table III).

Table III - List of suggestions

take in Table III

Furthermore an observer was present to record users' interaction with DLs and also to make sure each user understood terminologies used in the questionnaire and the purpose of each task given to them.

Data collected were analysed with the Statistical Package for the Social Sciences (SPSS), version 11.5. T-Test and Analysis of Variance (ANOVA) were applied to identify differences, and to test potential significant differences between the three user types. The significance value used for this study was $\mathrm{p}<0.05$.

\section{Results}

User Preferences for DLs

As mentioned above, the first part of the questionnaire that users had to complete following their interactions with the two DLs used in our experiment consisted of suggestions on the part of users themselves as to the features and functionality that they would like to see in DLs.

We have grouped user responses into four sections: Functionality, Interface, Content and Usability (Table IV) because these four issues are important for the development of DLs. Thus as far as functionality requirements are concerned, users indicated that, they are interested to receive as mush information as they can about materials that have searched for, as well as related information about them. Moreover, they would like to have access to other DLs from the original DL. This highlights that users' expectations of DLs are to get information they required directly or indirectly. Users' responses regarding interfaces of DLs were more about the way they could access links. Users prefer to use drop down menus or buttons rather than a simple link. Therefore they were concerned about seeing more visual interfaces than texts. The content of most DLs are focused on articles only. Also, users' opinion was that from DLs they should to be able to access other sources of information such as newspapers and e-books as well. Finally, their main usability concerns were more to do with using a powerful search engine and the help service offered by DLs.

Although, as expected, most of the suggestions obtained in this section came from intermediate and advanced users, the suggestions of novice users primarily targeted features such as: easy links to use for each function of the DL; back and forward buttons; and the ability to save searches for future references. Moreover, it is to be 
remarked that only advanced participants suggested being able to see feedback on materials (such as books, audio content) from other users, suggesting that only this category of users (advanced) is interested in pooling a range of opinions on the particular type of content being accessed.

Table IV - Users' suggestions on future DL's features

take in Table IV

\section{Requirements}

In the second part of the questionnaire administered to participants in our study, they had to prioritise 5 given requirements in order of 1-5 (1 being the most important and 5 the least important requirement). Accordingly, Figure 1 depicts the relative rankings of the five requirements. What is to be remarked is that t-tests revealed that there was general agreement $(\mathrm{df}=47, \mathrm{p}=0.000)$ among all users as regards their ranking opinions of each requirement. The overall picture (Figure 1) is that all users agree on the importance of finding information easily and quickly in DLs (R1) as well as being able to be familiarized with DLs (R2), have more priority than other requirements. Although experts (Blandford (2004) and D'Alessandro (2005)) have emphasised on collaborative knowledge working in DLs, our results seem to indicate that users think otherwise. Hence, in our study R5 (DL should allow users to discuss about documents to support collaborative knowledge working), was found to be the least important requirement, according to all three types of users considered.

What is also interesting to note is that, as Table $\mathrm{V}$ highlights (and an ANOVA confirms) is that, with one exception, the relative ranking of the 5 requirements does not depend on the IT proficiency of users. This shows that, in the particular case of 4 of the 5 requirements given in our study, the opinions of users were largely similar and independent of the IT background, which is thus not an effective differentiating factor here. It is only in the case of R2 (DLs should be designed in such a way to help those users who are not sure of what they are looking) that the results depended on the IT profile of the user $(\mathrm{p}=0.011, \mathrm{~F}=5.018)$; the importance of this requirement has a clear downward trend, with it being very much appreciated by novice users and almost totally discounted by advanced ones.

Figure 1 - Prioritised requirements by users

take in Figure 1

The mean value for all three types of users is close. According to these results, all users agree that being able to collaborate and discuss about documents in DLs is less important than the others (Table II). The same applies to getting positive effect on individuals' way of working with DLs.

take in Table $\mathrm{V}$

Table V - Prioritised requirements by each category 
In addition to what users said about their idea of an ideal DL, in the last part of the experiment users ranked 19 suggestions (Table III) for future DLs. T-test analysis revealed that there was general agreement among all users as regards their rankings for the given suggestions. These suggestions can be categorised in three groups of (Very important, important, not too important), based on their mean participantdefined importance level.

Very important suggestions:

- $\quad$ S18 - The DL should be easy to learn $(\mathrm{df}=47, \mathrm{p}=0.000$, mean value $=4.75)$;

- S13 - To be reliable in terms of finding result of a search ( $\mathrm{df}=47, \mathrm{p}=0.000$, mean value $=4.48$ );

- $\quad$ S15 - To categorise books based on their subject ( $\mathrm{df}=47, \mathrm{p}=0.000$, mean value $=4.21)$

- $\quad$ S16 - To give a list of most important resources available ( $\mathrm{df}=47, \mathrm{p}=0.000$; mean value $=4.12$ );

- S3 - A DL should be like a physical library where users can see all other books related to the same subject $(\mathrm{df}=47, \mathrm{p}=0.000$, mean value $=4.06)$;

Important suggestions:

- $\quad$ S1 - The DL should contain Music and Video as well as article and book ( $\mathrm{df}=$ $47, \mathrm{p}=0.000$, mean value $=3.79$ );

- S4 - All users should have a profile in that digital library website where they can see information such as: list of all articles and books they have saved, list of all music they listened to and videos watched $(\mathrm{df}=47, \mathrm{p}=0.001$, mean value $=3.58$;

- S8 - An alert should be available whenever a new item related to the user's subject is in the DL ( $d f=47, p=0.000$, mean value $=3.87$ );

- S14 - DL should have the picture of books' front cover beside other details of the book $(\mathrm{df}=47, \mathrm{p}=0.000$, mean value $=3.71)$;

- S19 - To get feedback from other readers $(\mathrm{df}=47, \mathrm{p}=0.000$, mean value $=$ $3.65)$;

Not too important suggestions:

- $\mathrm{S} 17$ - The DL should have frequent visited books available ( $\mathrm{df}=47, \mathrm{p}=$ 0.007 , mean value $=3.42$ );

- $\quad$ S6 - To see a list of all last printed or visited items from a DL ( $d f=47, p=$ 0.046 , mean value $=3.31$ );

- S12 - Users should be able to co-operate and communicate with other users of the DL $(\mathrm{df}=47, \mathrm{p}=0.041$, mean value $=2.67)$.

Moreover, an ANOVA test revealed that only S16 $(\mathrm{p}=0.027, \mathrm{~F}=3.923)$ and $\mathrm{S} 17(\mathrm{p}=$ $0.025, \mathrm{~F}=4.028)$ are depended on the IT profile of users. Thus, both advanced and novice users are keener about seeing a list of most important resources available (S16) in DLs than intermediate users. Since advanced users have more experience with DLs they have sense the need for a link to other resources. Also, our experience indicates that novice users, not very confident with using DLs, are afraid that they might not find what they need. Therefore, they feel that having access to other sources of information will give them a better chance of finding their sought-after item. This 
contrasts to intermediate users, who, although knowing how to use DLs, are not actually aware of the full functionality of DLs (and their limitations, consequently). In other words - intermediate users seem to over-estimate their DLs skills and abilities.

Although advanced and novice users are not too eager to see a list of frequent visited books (S17), they have nevertheless given this suggestion higher average ratings than intermediate users. Moreover, t-test analysis $(\mathrm{df}=15, \mathrm{p}=0.609$, mean value $=2.88)$ shows that there was general disagreement among intermediate users as regards S17. Thus, eight intermediate users believe S17 feature is little important, two users believe it is moderately important and six users think it is very important, with the majority of users from this category nevertheless not being in favour of this suggestion.

According to these results (Figure 2) the two most important features within DLs which all three types of users agree on is that DLs should be easy to learn (S18) and have reliable search engine (S13).

Figure 2 - Credited given to 19 given suggestions

take in Figure 2

A t-test analysis for novice users shows agreement among them and beside suggestions $\mathrm{S} 18(\mathrm{df}=15, \mathrm{p}=0.000$, mean value $=4.81)$ and $\mathrm{S} 13(\mathrm{df}=15, \mathrm{p}=0.000$, mean value $=4.56$ ) stated above, they gave high rankings (Figure 3 ) to features such as: S3 - being able to see a list of all other related books in a same subject ( $\mathrm{df}=15, \mathrm{p}$ $=0.000$, mean value $=4.44), \mathrm{S} 16$ - give a list of most important resources available $(\mathrm{df}=15, \mathrm{p}=0.000$, mean value $=4.44), \mathrm{S} 15$ - categorise books based on their subjects $(\mathrm{df}=15, \mathrm{p}=0.000$, mean value $=4.19), \mathrm{S} 1$ have music and video materials in DLs $(\mathrm{df}=15, \mathrm{p}=0.000$, mean value $=4.12)$, and $\mathrm{S} 4$ - all users should have a user profile in $\mathrm{DL}(\mathrm{df}=15, \mathrm{p}=0.000$, mean value $=4.00)$.

Moreover, there was agreement among these categories for less important features such as: $\mathrm{S} 19$ - get feedback from other readers $(\mathrm{df}=15, \mathrm{p}=0.001$, mean value $=$ 3.88), S17 - have a list of frequent visited books ( $\mathrm{df}=15, \mathrm{p}=0.005$, mean value $=$ 3.81 ), and S14 - have pictures of books' front cover ( $\mathrm{df}=15, \mathrm{p}=0.036$, mean value $=$ 3.69).

These results therefore indicate that novice users are more concerned about using DLs easily, search engine reliability, presented information accuracy, and also the contents of DLs, rather than getting additional information about materials from other users in DLs.

Figure 3 - Credit given to 19 suggestions from novice type

take in Figure 3

As opposed to novice users, intermediate users (Figure 4) think it is important to be informed of the availability of new materials in DLs (S8) and t-test analysis shows 
there is an agreement among them $(\mathrm{df}=15, \mathrm{p}=0.000$, mean value $=4.25)$. Similar to novice users, top of their preferences were suggestions $\mathrm{S} 18(\mathrm{df}=15, \mathrm{p}=0.000$, mean value $=4.75), \mathrm{S} 13(\mathrm{df}=15, \mathrm{p}=0.000$, mean value $=4.37)$, and $\mathrm{S} 15(\mathrm{df}=15, \mathrm{p}=$ 0.004 , mean value $=3.94)$.

Echoing the opinions of novice users, intermediate users indicated that $\mathrm{S} 14$ (df $=15, \mathrm{p}$ $=0.027$, mean value $=3.50$ ) is not a very important requirement of DLs. However as opposed to novice users, this category of users do not consider features $\mathrm{S} 3$ ( $\mathrm{df}=15, \mathrm{p}$ $=0.018$, mean value $=3.75), \mathrm{S} 16(\mathrm{df}=15, \mathrm{p}=0.046$, mean value $=3.63)$, and $\mathrm{S} 1(\mathrm{df}$ $=15, \mathrm{p}=0.041$, mean value $=3.50)$ as very important in DLs. Moreover, they agreed that the least important feature is $\mathrm{S} 12(\mathrm{df}=15, \mathrm{p}=0.046$, mean value $=2.38)$. Hence, the results of our survey revealed that time is an important issue for intermediate users, as users from this category indicated that they wished to spend less time in finding information in DLs. Moreover, they were not in favour of spending time communicating with other users while using DLs.

Figure 4 - Credit given to 19 suggestions from intermediate users

take in Figure 4

Advanced users (Figure 5) also awarded high-rankings to $\mathrm{S} 18$ ( $\mathrm{df}=15, \mathrm{p}=0.000$, mean value $=4.69), \mathrm{S} 13(\mathrm{df}=15, \mathrm{p}=0.000$, mean value $=4.50), \mathrm{S} 15(\mathrm{df}=15, \mathrm{p}=$ 0.000 , mean value $=4.50), \mathrm{S} 16(\mathrm{df}=15, \mathrm{p}=0.000$, mean value $=4.31)$, and S3 $(\mathrm{df}=$ $15, \mathrm{p}=0.000$, mean value $=4.00)$.

Similar to novice users, this category did not give high priorities to $\mathrm{S} 17$ ( $\mathrm{df}=15, \mathrm{p}=$ 0.034 , mean value $=3.56)$ and $\mathrm{S} 19(\mathrm{df}=15, \mathrm{p}=0.013$, mean value $=3.75)$. However, as opposed to novice and intermediate users, advanced users considered S14 (df $=15, p=0.001$, mean value $=3.94)$ to be an important feature in DLs, whereas $\mathrm{S} 1(\mathrm{df}=15 ; \mathrm{p}=0.009$, mean value $=3.75)$, and $\mathrm{S} 8(\mathrm{df}=15, \mathrm{p}=0.006$, mean value $=3.75$ ) are less important. Another feature not very highly thought of by advanced users is S7 - to have a list of all related subject to the last attended ones by a user $(\mathrm{df}=15, \mathrm{p}=0.045$, mean value $=3.56)$.

Almost all participants in the advanced users category were researchers who used DLs frequently. These users felt the need to have the picture of the front cover of books in DLs, thus augmenting textual information with graphical content. Surprisingly, this experienced group of users prefers to get text materials in DLs than audio and video. Furthermore they are not in favour of being informed of new materials in DLs as much as intermediate users do. This could mean that because of their frequent access to DLs, they know how to search for items they need. However, as can be seen in Figures 3- 5, although advanced users have more experience with DLs than the other two categories, they do share broadly similar thoughts about the 19 suggestions put forward in our survey.

Figure 5 - Credit given to 19 suggestions from advanced users take in Figure 5 


\section{Conclusion}

This paper has explored users' requirements of DLs. As opposed to previous work (Stelmaszewska and Blandford, forthcoming; Stelmaszewska, 2001; Theng et al., 2000) in the area, which has mainly focused on usability issues in DLs, or on specialist users, in our work we have elicited user opinions regarding features that DLs should have from users with a wider spectrum of IT skills, ranging from the novice to the advanced users.

Users suggested that DLs be easy to learn and reliable in terms of obtaining search results. DL designers must therefore consider that for users being able to access reliable information easily and fast is more important than the layout and interfaces of DLs. Surprisingly, users were not too keen on reading e-books in DLs, but wished instead to see information about books being made available in DLs. However, users expect DLs: to contain more information about available books; to categorise them based on their subject matter; to have pictures of the front cover of the book displayed alongside other details of the book; to display all other books related to the searched book, as well as a list of most frequently visited books. Hence, our results would seem to indicate that as far as books are concerned, DLs still have outstanding issues, if the self-expressed user desire is to be taken into account.

Our experiment also shows that users are not too keen about being able to co-operate and communicate with other DL users. Novice users were more in favour of having a user profile on DLs than the other two categories (advanced and intermediate). Surprisingly, having an online help assistant was also one of the least important suggestions from both the novice and advanced users' point of view.

Although this experiment was a first step towards the design of a DL based on what users want, it has clearly highlighted the need to improve the current status and experience users have with DLs, if DLs are to meet users' needs for quick access to information. For this, it is essential that user requirements form an integral part in the design and development part user-centred DLs, and this forms the focus of our future work.

\section{Acknowledgements}

We are grateful to all 48 participants who took part in this study and to Stephen Gulliver for his comments on the paper.

\section{References}

ADAMS, A. and BLANDFORD, A., 2002. Digital libraries in academia: challenges and changes, E. LIM, S. FOO, KHOO, CHRISTOPHER S. G., H. CHEN, E.A. FOX, S.R. URS and C. THANOS, eds. In: Digital Libraries: People Knowledge and Technology. 5th International Conference on Asian Digital Libraries, ICADL 2002. Proceedings, 11-14 December 2002, December 11 - 142002 2002, Springer-Verlag pp392-403. 
BLANDFORD, A., forthcoming. Interacting with information resources: digital libraries for education. http://www.uclic.ucl.ac.uk/annb/DLUsability/DLindex.html. University College London: International Journal of Learning Technologies.

BLANDFORD, A., 2004. Understanding user's experience: evaluation of digital libraries, M. AGOSTI and N. FUHR, eds. In: Workshop on the Evaluation of Digital Libraries, October 2004, DELOS Workshop pp31-34.

BLANDFORD, A. and BUCHANAN, G., 2003. Usability of digital libraries: a source of creative tensions with technical developments. http://www.ieeetcdl.org/Bulletin/summer2003/blandford/blandford.html. University College London University: TCDL.

BLANDFORD, A., KEITH, S., CONNELL, I. and EDWARDS, H., 2004. Analytical Usability Evaluation for Digital Libraries: a Case Study, H. CHEN, H. WACTLAR and C. CHEN, eds. In: Proceedings of the 4th ACM/IEEE-CS joint conference on Digital libraries, 7-11 June 2004, ACM/IEEE pp27-36.

BRYAN-KINNS, N. and BLANDFORD, A., July 2000. A survey of user studies for digital libraries. RIDL Working paper. United Kingdom.

BRYAN-KINNS, N., BLANDFORD, A. and THIMBLEBY, H., 2000. Interaction Modelling for Digital Libraries. http://www.uclic.ucl.ac.uk/annb/docs/eims.pdf. Middlesex University, United Kingdom.

D'ALESSANDRO, D.M. and D'ALESSANDRO, M.P., 2005. Initial Experiences in developing a chronologically organised digital library for continuing education in Biodefense. http://www.dlib.org/dlib/april05/dalessandro/04dalessandro.html. Iowa City, IA: D-Lib Magazine.

FIELDS, B., KEITH, S. and BLANDFORD, A., 2004. Designing for Expert Information Finding Strategies, S. FINCHER and P. MARKOPOULOS, eds. In: People and Computers XVIII - Design for Life, 2004, Springer pp89-102.

FOX, E.A., HIX, D., NOWELL, L.T., BRUENI, D.J., WAKE, W.C. and HEATH, L.S., 1993. Users, User Interfaces, and Objects: Envision, a Digital Library. Journal of the American Society for Information Science, 44(8), pp. 480-491.

HSIEH-YEE, I., 1993. Effects of Search Experience and Subject Knowledge on the Search Tactics of Novice and Experienced Searchers. Journal of the American Society for Information Science, 3(44), pp. 161-174.

JONES, S., MCINNES, S. and STAVELEY, M.S., 1999. A graphical user interface for Boolean query specification. International Journal on Digital Libraries, 2(2/3), pp. 207-223.

LANG, B., 1998. Developing the digital library. In: L. CARPENTER, S. SHAW and A. PRESCOTT, eds, Towards the Digital Library. 1. London: The British Library, pp. 227-233. 
LEINER, B.M., 31 May 2005, 2005-last update, the scope of the digital library [Homepage of wikipedia], [Online]. Available: http://en.wikipedia.org/wiki/Digital_library [12 June, 2005].

SLONIM, J. and BARONLAURIE, L., 1994. Networked Information Systems As Digital Libraries. In: N.R. ADAM, B.K. BHARGAVA and Y. YESHA, eds, Digital Libraries. 1. Berlin Heidelberg New York: Springer-Verlag, pp. 51-61.

STELMASZEWSKA, H. and BLANDFORD, A., forthcoming. From Physical to digital: a case study of computer scientists' behaviour in physical libraries. International Journal on Digital Libraries, 4.2, pp. 82-92.

STELMASZEWSKA, H., BLANDFORD, A. and BRYAN-KINNS, N., 2001. Use of multiple digital libraries: a case study, E.A. FOX and C.L. BORGMAN, eds. In: JCDL 2001, 24 June 2001, ACM pp179-188.

SULEMAN, H. and FOX, E.A., 2001. A Framework for building open digital libraries. http://www.dlib.org/dlib/december01/suleman/12suleman.html. Virginia Tech: D-Lib Magazine.

THENG, Y.L., MOHD-NASIR, N. and THIMBLEBY, H., 2000. Purpose and Usability of Digital Libraries. ACM DL, 1(58113-231), pp. 1-236.

TWIDALE, M.B. and NICHOLS, D.M., 1998. Using studies of collaborative activity in physical environments to inform the design of digital libraries. Digital Library. CSCW'98 Workshop, Collaborative and co-operative information seeking in digital information environments.

WITTEN, I.H., BAINBRIDGE, D. and BODDIE, S.J., 2001. Power to the People: End-user Building of Digital Library Collections, 24-28 June 2001, ACM.

ZHANG, J. and MARCHIONINI, G., 2005. Evaluation and evolution of a browse and search interface: relation browser, L. DELCAMBRE and G. GIULIANO, eds. In: Proceedings of the 2005 national conference on Digital government research, 15-18 May 2005, Digital Government Research Center pp179-188. 